\title{
Gyrokinetic Simulations of Spherical Tokamaks
}

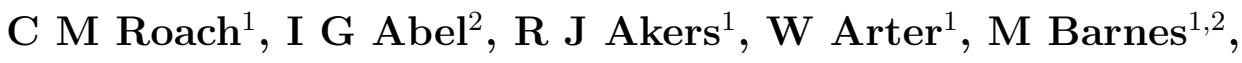 \\ Y Camenen ${ }^{3}$, F J Casson ${ }^{3}$, G Colyer ${ }^{1}$, J W Connor ${ }^{1}$, \\ S C Cowley ${ }^{1}$, D Dickinson ${ }^{4}$, W Dorland ${ }^{5}$, A R Field ${ }^{1}$, \\ W Guttenfelder ${ }^{3}$, G W Hammett ${ }^{6}$, R J Hastie ${ }^{1}$, E Highcock ${ }^{2}$, \\ N F Loureiro ${ }^{1}$, A G Peeters ${ }^{3}$, M Reshko ${ }^{4}$, S Saarelma ${ }^{1}$, \\ A A Schekochihin ${ }^{2}, \mathrm{M}$ Valovic $^{1}$ and $\mathrm{H} \mathbf{R}$ Wilson $^{4}$ \\ ${ }^{1}$ EURATOM/UKAEA Fusion Association, Culham Science Centre, Abingdon, \\ Oxfordshire, OX14 3DB, UK \\ 2 Rudolf Peierls Centre for Theoretical Physics, University of Oxford, 1 Keble Road, \\ Oxford OX1 3NP, UK \\ ${ }^{3}$ CFSA, Department of Physics, University of Warwick, Coventry CV4 7AL, UK \\ 4 Department of Physics, University of York, Heslington, York, YO10 5DD, UK \\ ${ }^{5}$ University of Maryland, College Park, Maryland, USA \\ ${ }^{6}$ Princeton Plasma Physics Laboratory, Princeton University, New Jersey, USA
}

\begin{abstract}
This paper reviews transport and confinement in spherical tokamaks (STs) and our current physics understanding that is partly based on gyrokinetic simulations. We show that equilibrium flow shear can sometimes entirely suppress ion scale turbulence in today's STs. Advanced nonlinear simulations of electron temperature gradient (ETG) driven turbulence, including kinetic ion physics, collisions and equilibrium flow shear, support the model that ETG turbulence can explain electron heat transport in many ST discharges.
\end{abstract}

\section{Introduction}

Data from the 1MA-scale experiments MAST and NSTX are unveiling the nature of plasma confinement in spherical tokamaks (STs) $[1,2]$. Neutral beam injection (NBI) drives strong toroidal flow in these devices, and radial shear in the component perpendicular to the magnetic field acts to twist the turbulent eddies that may be responsible for anomalous transport. Perpendicular flow shear is characterised by the parameter, $\gamma_{E}=d V_{\perp} / d r$, where $1 / \gamma_{E}$ corresponds to the time for an eddy to be tilted by $45^{\circ}$ in the plane perpendicular to the magnetic field. This twisting of turbulent eddies competes with and potentially disrupts the linear instability drive, and when $\gamma_{E}$ exceeds the linear growth rates, it can suppress turbulence $[3,4]$. The parallel component of the sheared toroidal flow, on the other hand, provides a linear drive that can enhance the turbulence. We show that equilibrium geometry at mid-radius in STs favours the suppression of turbulence from the perpendicular component of the sheared toroidal flow. In Section 2 we review our current physics understanding of plasma transport in STs, and highlight the important role of equilibrium flow shear in regulating the more slowly growing longer wavelength modes. Equilibrium flow shear can be accommodated in the gyrokinetic framework, as outlined in Section 3, and has recently been implemented and tested in local gyrokinetic codes, GS2 [5] and GKW [6]. Linear gyrokinetic simulations 
demonstrate that, at mid-radius in MAST, the typical experimental level of $\gamma_{E}$ stabilises ITG modes, and leaves the faster growing ETG modes unstable. Section 4 assesses the influence of flow shear on nonlinear simulations of ETG turbulence that have predicted significant electron heat transport in MAST [7], and probes the robustness of that important result using more sophisticated gyrokinetic models that include flow shear, and which also include kinetic ions and collisions. A summary of the main conclusions is presented in Section 5.

\section{Confinement, Transport and Gyrokinetic Studies of STs}

The measured energy confinement time, $\tau_{E}$, in MAST and NSTX is broadly consistent with the ITER IPB98 $(y, 2)$ scaling law $\tau_{E}=0.0562 I_{p}^{0.93} B_{\phi}^{0.15} P_{L}^{-0.69} n^{0.41} M^{0.19} R^{1.97}(a / R)^{0.58}$ $\kappa^{0.78}[8]$ derived from conventional aspect ratio devices, where $\tau_{E}$, plasma current $I_{p}$, toroidal field $B_{\phi}$, loss power $P_{L}$, density $n$, mean ion mass number $M$, and major radius $R$ are in units $\mathrm{s}, \mathrm{MA}, \mathrm{T}, \mathrm{MW}, 10^{19} \mathrm{~m}^{-3}$, AMU, and $\mathrm{m}$ respectively, $a / R$ is inverse aspect ratio and $\kappa$ is elongation. Detailed parameter scans, however, indicate that confinement scales differently in STs [2,9], and the MAST scaling in terms of engineering parameters, $\tau_{E}=0.186 I_{p}^{0.59} B_{\phi}^{1.4} P_{L}^{-0.73}$ depends more weakly on $I_{p}$ and more strongly on $B_{\phi}[9]$. This scaling, favourable for future ST devices, is not yet understood. NSTX finds a similar scaling and attributes the improvement of $\tau_{E}$ with: (i) increasing $B_{\phi}$ to a reduction of transport in the (dominant) electron channel; and (ii) increasing $I_{p}$ to falling neoclassical ion heat transport [2]. We note that the impact of strong toroidal flows on energy confinement is not clearly isolated in these scaling laws- and perhaps it should be. In any tokamak plasma heated by toroidally injected NBI (like MAST and NSTX), toroidal momentum and energy confinement times can be shown to be related via: $\tau_{\phi} \sim M_{\phi}\left(v_{b} / v_{t i}\right) \tau_{E}$, where $M_{\phi}=V_{\phi} / v_{t i}$ is the toroidal Mach number, $V_{\phi}$ is the toroidal flow, and $v_{b}, v_{t i}$ are the velocities of beam and thermal ions respectively. In MAST $M_{\phi} \sim 0.4$ and $v_{b} / v_{t i} \sim \sqrt{60}$, which implies $\tau_{\phi} \sim 3 \tau_{E}$. The fact that $\tau_{\phi}$ is determined by ion confinement suggests that, for these MAST conditions, the energy losses are predominantly through the electron channel.

Both MAST and NSTX have frequently observed the suppression of anomalous ion heat transport, with the measured ion thermal diffusivity $\chi_{i}$ approaching the neoclassical prediction $\chi_{i}^{n c}$, while the experimental electron thermal diffusivity $\chi_{e}$ is usually much larger $[2,10]$. Analysis of ion channel internal transport barriers in co-NBI MAST plasmas suggests that these arise owing to the sheared flow suppression of ITG turbulence [10]. Transport analysis of similar L- and H-mode MAST plasmas [10] revealed for $r / a>0.5$ : $\chi_{i} \sim \chi_{i}^{n c}$ and $\chi_{e} \sim(2-4) \chi_{i}^{n c}$ in H-mode; however, both $\chi_{i}, \chi_{e}>\chi_{i}^{n c}$ in L-mode. $\ddagger$ The Trapped-Gyro-Landau Fluid model (TGLF) for anomalous transport [12] is applicable to shaped low $R / a$ plasmas, and first comparisons with MAST and NSTX data [13] also suggest that sheared flow plays a vital role in stabilising ITG turbulence. For the MAST and NSTX plasmas analysed, TGLF predicts $\chi_{i} \sim \chi_{i}^{n c}$ over a significant fraction of the minor radius, and that ETG turbulence dominates electron heat flux $q_{e}$ for $q>1$, but

$\ddagger$ Data from the MAST discharges in [10] are available through the 2008 public release of the ITPA international multi-tokamak confinement profile database [11]. 
overestimates $T_{e}$ in the core. In the MAST L-mode discharge \#8505 [10], where ion heat transport was anomalous, ITG turbulence was modelled, without flow shear $\S$, using the global gyrokinetic particle-in-cell code ORB5 [14]. In the gradient zone the nonlinearly saturated $\chi_{i}$ was found to be significantly larger than the measured value, indicating the importance of including flow shear in the modelling of ion scale turbulence in MAST.

Gyrokinetic simulation for STs faces a number of challenges: (i) the gyrokinetic expansion parameter $\delta=\rho_{i} / L$ is not very small ( $L$ is the equilibrium length scale and $\rho_{i} / L \sim 0.02$ in MAST); (ii) highly shaped low aspect ratio plasmas rule out simple analytic equilibrium models; (iii) magnetic perturbations can be important if $\beta$ is large; (iv) strong toroidal flows with $M_{\phi} \sim 1$; (v) radially sheared flows impact on microturbulence; and (vi) there can be significant populations of super-Alfvénic fast particles. Gyrokinetics codes are rising to meet many of these challenges: Appendix A demonstrates close agreement in electrostatic microstability results from three local flux-tube gyrokinetic codes (GKW, GS2 and GYRO [15]) for a typical strongly shaped MAST equilibrium flux surface. Microinstability based transport mechanisms in STs have been explored using linear studies (mainly with GS2 and GYRO). The effect of including $\delta \boldsymbol{B}$ was found, using GS2, to be significant at mid-radius in MAST $[16,17]$, where locally $\beta \sim 0.1$ : at $k_{\perp} \rho_{i} \lesssim 1$, where $k_{\perp}$ is the perpendicular wavenumber, electromagnetic modes dominated over ITG modes, and these exhibited tearing and twisting parity in the H-mode and L-mode plasmas respectively; furthermore including $\delta B_{\|}$reduced the growth rates of ETG instabilities [17]. A detailed study of microtearing modes found that they are not peculiar to the ST and that their growth rate is sensitive to $d T_{e} / d r$, magnetic drifts, and $\nu_{* e}[18]$. Microtearing modes have since been found in conventional aspect ratio tokamak plasmas $[19,20]$, and a nonlinear microtearing mode saturation theory estimate of $\chi_{e}$ was found to be consistent with NSTX measurements [21]. Electron heat transport was observed to increase dramatically with rising beam power in the core of NSTX, where fast particle instabilities were observed and thermal temperature gradients were too low to drive transport via the usual mechanisms [22]. Microtearing modes, fast particle instabilities, and sonic flows will not be addressed further in this paper.

GS2 microstability analyses of MAST discharges, without flow shear, have revealed that the sheared flow stabilisation criterion $\gamma_{E}=r / q d\left(q v_{E} / r\right) / d r>\gamma_{\max }$ [4] (where $v_{E}$ is the equilibrium $E \times B$ velocity and $\gamma_{\max }$ is the maximum linear growth rate) is often satisfied for ITG but not ETG modes in MAST plasmas [16, 17]. Nonlinear calculations for STs have therefore generally focussed on modelling ETG turbulence. GS2 simulations (without flow shear) for a typical H-mode plasma, suggest that ETG can carry significant electron heat flux at mid-radius in MAST [7], consistent with findings from GYRO simulations for NSTX [2]. In this paper flow shear will be included self-consistently in local gyrokinetic simulations, to assess more thoroughly the impact of flow shear on ITG and ETG turbulence in MAST.

$\S$ The TGLF model predicts that flow shear partly suppresses ITG turbulence in this plasma. 


\section{Gyrokinetics with Equilibrium Flow Shear}

We now describe how equilibrium flow shear can be accommodated in the gyrokinetic equation (GKE) that describes microinstabilities. The GKE for the leading order, nonadiabatic piece of the perturbed guiding centre distribution, $g$, with sonic toroidal flows $\boldsymbol{V}=R \Omega(\psi) \boldsymbol{e}_{\boldsymbol{\phi}}$ (where the toroidal frequency $\Omega$ is a function of poloidal flux $\psi$ ) appears as equation (56) of [23]. We assume the toroidal flow contribution dominates the radial electric field, and take $V=R \Omega(\psi) \sim O\left(M_{\phi} v_{t i}\right)$. Expanding to leading order in $M_{\phi}$ (where $\rho_{i} / L<M_{\phi}<1$ ) while retaining locally steep radial rotation gradients by ordering $\partial \boldsymbol{V} / \partial r=R^{2} B_{p} \Omega^{\prime} \boldsymbol{e}_{\boldsymbol{\phi}} \sim O\left(v_{t i} / L\right.$ ) (where ' denotes $d / d \psi$ ), and dropping nonlinear and magnetic perturbation terms for brevity, yields the linear electrostatic GKE with subsonic sheared toroidal flow:

$$
\begin{aligned}
& \left(\frac{\partial}{\partial t}+V \cdot \nabla_{\perp}\right) g+\left(c_{\|} \boldsymbol{b}+\boldsymbol{c}_{\boldsymbol{D} \mathbf{0}}\right) \cdot \nabla_{R} g=-\frac{q J_{0}}{m} \frac{\partial F}{\partial \epsilon}\left(\frac{\partial}{\partial t}+V \cdot \nabla_{\perp}\right) \tilde{\Phi} \\
& -\frac{q J_{0}}{m \Omega} \boldsymbol{b} \times \nabla_{R} \tilde{\Phi} \cdot \nabla_{R} F+\frac{q J_{0}}{m \Omega} \frac{\partial F}{\partial \epsilon} b \times \nabla_{R} \tilde{\Phi} \cdot\left[c_{\|} R \Omega^{\prime} \frac{B_{\phi}}{B} \nabla \psi\right]
\end{aligned}
$$

where the guiding centre velocity $\boldsymbol{v}=\boldsymbol{V}+\boldsymbol{c}, \boldsymbol{c}_{\boldsymbol{D} \mathbf{0}}=\boldsymbol{b} / \Omega \times\left(c_{\perp}^{2} / 2 \boldsymbol{\nabla} \log B+c_{\|}^{2} \boldsymbol{b} . \boldsymbol{\nabla} \boldsymbol{b}\right)$ contains the magnetic drifts, $\boldsymbol{V}=\left(R \Omega_{0}+R \Omega^{\prime}\left(\psi-\psi_{0}\right)\right) \boldsymbol{e}_{\boldsymbol{\phi}}, F=A(\psi) e^{-m \epsilon / T(\psi)}$ is the isotropic equilibrium distribution function in the co-moving frame, and $\epsilon=c_{\|}^{2} / 2+c_{\perp}^{2} / 2$. Terms in grey are due to sheared toroidal flow. The $\boldsymbol{V} \cdot \boldsymbol{\nabla}_{\perp}$ terms act to differentially advect perturbations. In a local flux tube, $\Omega_{0}$ appears as a Doppler shift in the perturbation frequency, and in codes, like GS2 and GKW, where FFTs are used in the radial direction $(x)$, the radial shearing term is implemented by forcing $k_{x}$ to depend linearly on time [24]. (Equivalently in ballooning space the shearing term is implemented by making the ballooning parameter $\theta_{0}$ depend linearly on time.) The final term on the RHS is a linear drive from the parallel component of the sheared equilibrium toroidal rotation. The shearing term is implemented via the equilibrium flow shearing rate, $\gamma_{E}=\frac{\rho}{q} \frac{d \Omega}{d \rho}$, where $\rho$ is the radial flux surface label. The linear equilibrium drive term is fully determined by $\gamma_{E}$ and is proportional to:

$$
-\frac{c_{\|}}{v_{t j}^{2}} \frac{q R B_{\phi}}{\rho B} \gamma_{E} \sim-\frac{c_{\|}}{v_{t j}^{2}} \frac{q R}{\rho} \gamma_{E} \text { at large aspect ratio }
$$

where $v_{t j}$ is the thermal velocity of species $j$. The implementation of the linear drive term in GS2 shows excellent agreement with analytic theory for the radially sheared parallel flow instability in slab geometry without magnetic shear.

\subsection{Linear Impact of Flow Shear on MAST}

Linear gyrokinetic microstability calculations for a typical mid-radius flux-surface in MAST were performed in ballooning space using GS2, and here we indicate how effective growth rates were obtained. At finite $\gamma_{E}$, a linear eigenfunction cannot strictly be represented by a ballooning mode with a specific single choice of the ballooning parameter $\theta_{0}$, as flow shear couples modes with different $\theta_{0}$ that can only be considered to be independent for $\gamma_{E}=0$. With infinitesimal flow shear, the true linear eigenmode can 

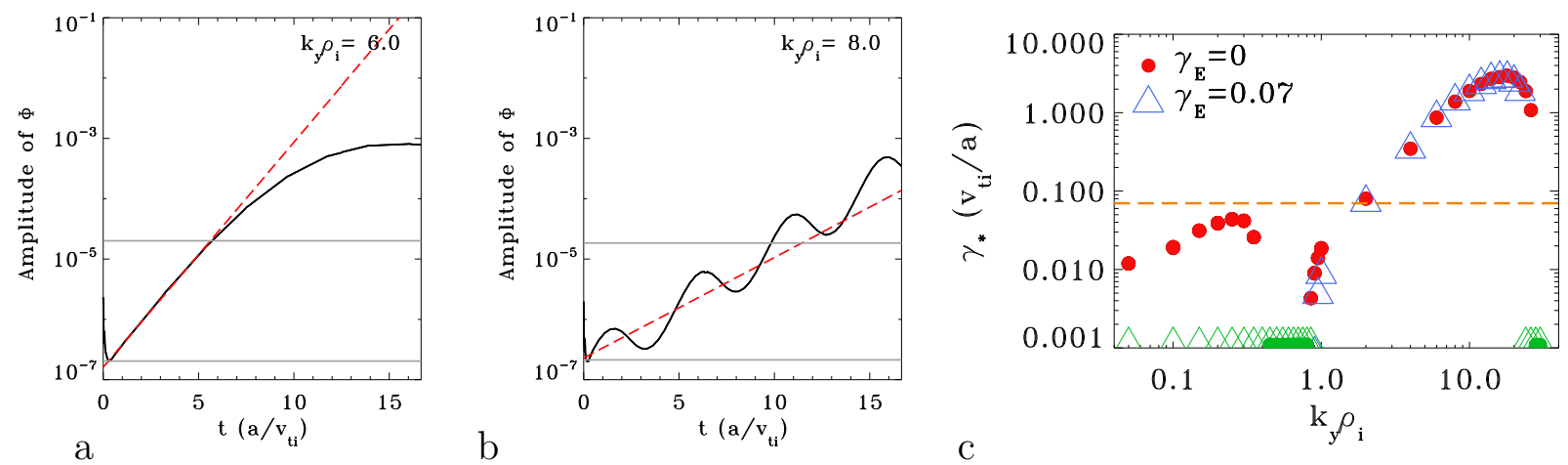

Figure 1. $\Phi$ amplitude versus $\mathrm{t}$ in linear electrostatic calculations for a mid-radius surface in MAST for (a) $k_{y} \rho_{i}=6, \gamma_{E}=0.07$ and (b) $k_{y} \rho_{i}=8, \gamma_{E}=0.5$. Horizontal grey lines indicate the amplitude range used to obtain the effective growth rate $\gamma_{*}$. (c) $\gamma_{*}$ versus $k_{y} \rho_{i}$ for $\gamma_{E}=0$ (circles) and $\gamma_{E}=0.07 a / v_{t i}$ (triangles). The horizontal dashed line shows the level of $\gamma_{E}$. Symbols at the $\gamma$-axis minimum denote stable modes.

be constructed as an ensemble of ballooning modes and has growth rate $\langle\gamma\rangle_{\theta_{0}}$, which is the $\theta_{0}$ averaged growth rate in the absence of flow shear [25]. It is still, however, useful to consider a pure ballooning mode with specified $\theta_{0}$, and its amplitude will grow as $e^{\left(\langle\gamma\rangle_{\theta_{0}}+C(t)\right) t}$, where $C(t)$ is oscillatory. An example of such growth is illustrated in Figure 1(b). Linear theory is only ever applicable up to the finite amplitude where nonlinear physics becomes important, and from any initial state with finite amplitude turbulence due to noise, this nonlinear threshold may be reached after a finite mode amplification. The crucial point here is that, even when the true linear eigenmode is stable for $\gamma_{E}<\gamma_{\max }$, a pure ballooning mode with specified $\theta_{0}$ can experience many linear growth times and reach the nonlinear threshold before the ballooning parameter is affected by flow shear.

It is straightforward to specify a prescription for obtaining an effective linear growth rate $\gamma_{*}$ for a pure ballooning mode with a particular choice of $k_{y}$. In an initial value code like GS2, it is sufficient to track the amplitude of the mode, following the associated evolution in $\theta_{0}(t)$. We adopt the following simple model to determine the effective linear growth rate $\gamma_{*}$ : (i) specify a finite mode amplification factor $F_{N L}$ (here we set $F_{N L}=100$ ), (ii) when/if a mode becomes amplified by $F_{N L}$, we consider it unstable and ignore subsequent linear evolution, (iii) determine $\gamma_{*}$ by fitting $\Phi\left(k_{y}, \theta_{0}(t), t\right)$ over the range of $t$ that achieved the required mode amplification, starting from an initial minimum in the amplitude. This procedure is illustrated in Figures 1 (a) and (b), and was followed to obtain the mid-radius MAST growth rate spectra in Figure 1(c) for $\gamma_{E}=0,0.07 v_{t i} / a$.

Figure 1(c) clearly illustrates, using linear results from GS2, that ITG modes $\left(k_{y} \rho_{i}<1\right)$ at mid-radius in MAST can be stabilised by a conservative level of $\gamma_{E}$, while more rapidly growing ETG modes remain unstable.

\subsection{Nonlinear ITG Simulations with Flow Shear for Large Aspect Ratio Plasmas}

Nonlinear calculations of ITG turbulence assuming adiabatic electrons (AE) and including equilibrium flow shear, have been carried out with GS2 and GKW both for circular

\| A related discussion for gyrofluid calculations with flow shear in ballooning space is given in [26]. 

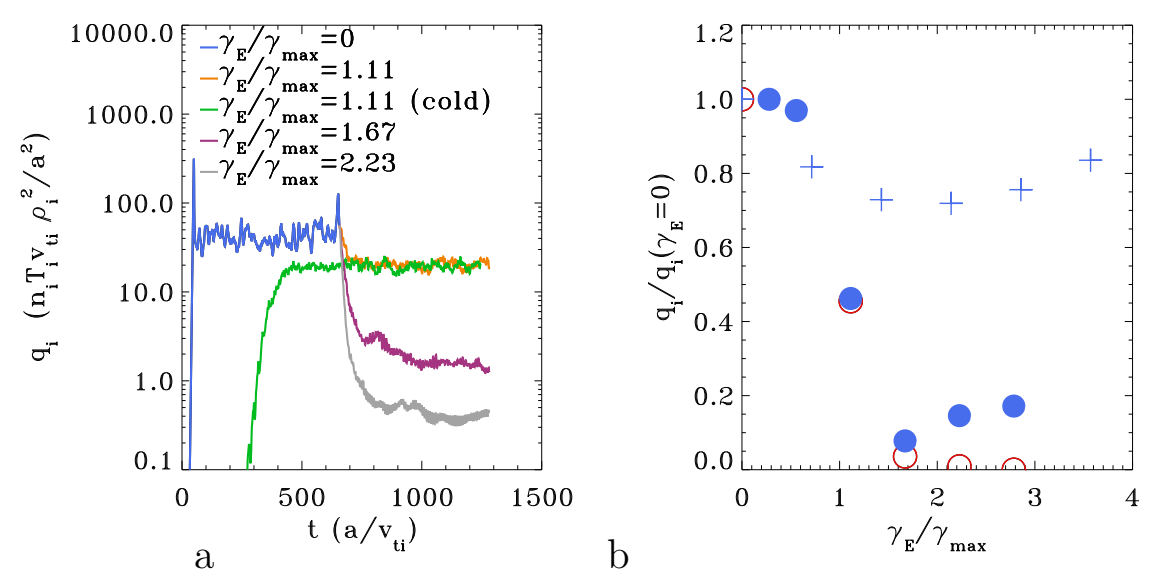

Figure 2. (a) Electrostatic GS2 simulations for the Cyclone parameters described for various levels of $\gamma_{E} / \gamma_{\max }$, without the flow shear linear drive term, showing $q_{i}$ versus t. In (b) the ratio of saturated $q_{i}$ to that with $\gamma_{E}=0$ is plotted versus $\gamma_{E} / \gamma_{\max }$ : circles show GS2 results for the Cyclone equilibrium described with (solid) and without (open) the shear flow linear drive term; and GKW results with the shear flow linear drive term for the standard case of [27] (crosses).

Cyclone [28] plasma equilibrium parameters $(R / a=3, r / a=0.54, q=1.39, \hat{s}=$ $\left.(r / q) d q / d r=0.8, \beta=\beta^{\prime}=0, R / L_{T_{i}}=6.9, R / L_{n}=2.2, T_{i}=T_{e}\right)$ and for the slightly different standard case of [27] with $q=2, \hat{s}=1.0$ and $r / a=0.5$. These simulations included 16 modes in the binormal perpendicular wavenumber that span $0.05<k_{y} \rho_{i}<0.75$, and were carried out for different levels of $\gamma_{E}$, both with and without the linear drive term. Simulations with finite $\gamma_{E}$ were performed either: (i) as warmstarts from the saturated state of the $\gamma_{E}=0$ simulation, or (ii) as cold-starts from low amplitude noise. The same saturated ion heat flux $q_{i}$ was reached in either scenario, as illustrated for $\gamma_{E} / \gamma_{\max }=1.11$ in Figure 2(a). GS2 results in Figure 2(b) show that without the linear drive term, $q_{i}$ is increasingly suppressed as the flow shear increases in strength, and becomes fully suppressed close to $\gamma_{E} / \gamma_{\max } \sim 2$ (as was found in [27]). When the linear drive is included, at low $\gamma_{E}, q_{i}$ falls with increasing flow shear to a minimum where it is not fully suppressed, and starts to increase with $\gamma_{E}$ above $\gamma_{E} / \gamma_{\max } \sim 1.5$ due to the additional linear drive that enhances the ITG growth rate [29]. GKW results in Figure 2(b) confirm that turbulence suppression is extremely modest for the standard case parameters of [27], where the linear drive term is stronger due to the larger factor $q R / r=12$ (N.B. $q R / r=7.7$ for the Cyclone equilibrium). At mid-radius in MAST this geometric factor is smaller: $q R / r \sim 4$. Given that the linear drive is reduced at lower values of $q R / r$ and $B_{\phi} / B$, shear flow suppression of turbulence is favoured by geometry at mid-radius in STs, where and the component of toroidal flow along the magnetic field is smaller than in conventional devices.

\section{Improved Nonlinear ETG Simulations for MAST}

Figure 1(c) showed that, at mid-radius in MAST, ITG modes are linearly suppressed by flow shear, while ETG modes are unaffected : we now explore the significance of ETG turbulence. Fully electromagnetic simulations with GS2, neglecting $\gamma_{E}$, demonstrated 
that the electron heat flux from ETG turbulence can be experimentally significant at midradius in MAST [7]. The reference model in [7] assumed adiabatic ions (AI), no collisions, spanned $0.01<k_{y} \rho_{e}<0.31$ with 32 modes, ran to $t \sim 300 a / v_{t e}$, and used plasma parameters: $\rho=\psi_{n}=0.4, r / a=0.566, r / R=0.35, q=1.35, \hat{s}=(\rho / q)(d q / d \rho)=0.29$, $\beta_{e}=0.049, T_{e}=0.59 \mathrm{keV},\left(1 / T_{e}\right) d T_{e} / d \rho=-2.04, n_{e}=4.4 \times 10^{19} \mathrm{~m}^{-3},\left(1 / n_{e}\right) d n_{e} / d \rho=0$. The predicted $\chi_{e}$ values with a range of domain sizes and resolutions, with/without kinetic ions (KI) and electron collisions, were consistent to within a factor around two [7]. Here we probe further the robustness of this very important conclusion with longer GS2 simulations that include kinetic ions, collisions and equilibrium flow shear. Despite the large impact of magnetic perturbations (especially $\delta B_{\|}$) on linear ETG growth rates [17], the saturated electron heat fluxes $q_{e}$ in electrostatic and electromagnetic simulations were similar and predominantly electrostatic $[7,30]$. For reasons of economy with computing resources, most of the GS2 simulations reported here are electrostatic, although a subset of key results were checked and found to be consistent with fully electromagnetic simulations.

a
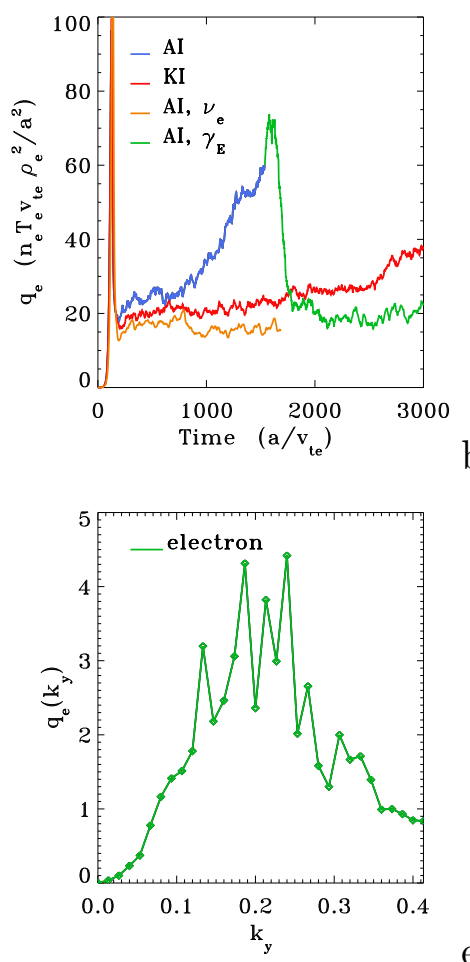

$\mathrm{b}$

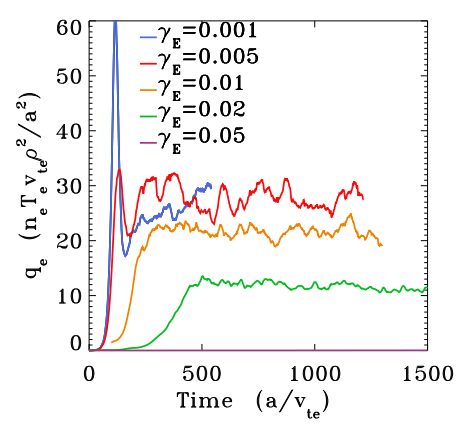

$\mathrm{c}$
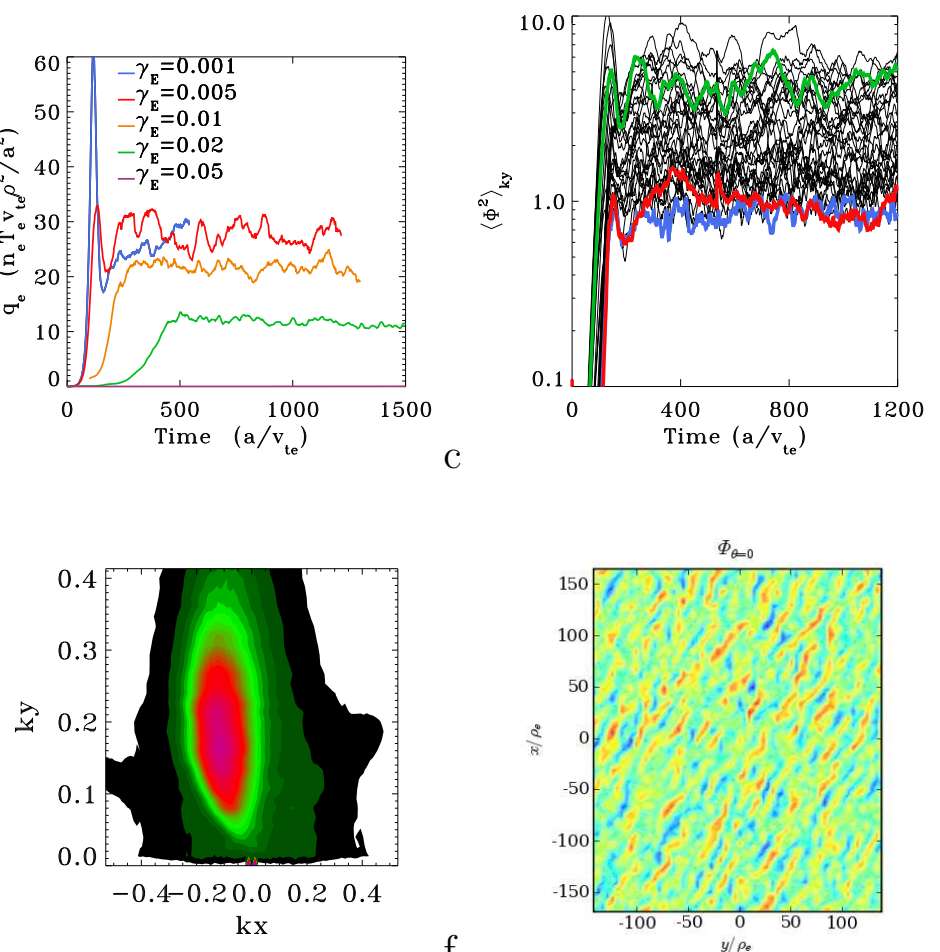

$\mathrm{e}$

Figure 3. (a) $q_{e}$ versus $t$ with collisionless AI and KI, AI with collisions, and collisionless AI with $\gamma_{E}=0.005$. (b) $q_{e}$ versus $t$ for various $\gamma_{E}$ for the Miller equilibrium with $L_{y}=75 \rho_{e}$ and $J_{\text {twist }}=4$. For the $\gamma_{E}=0.005$ case in (b): (c) $\left\langle\Phi^{2}\left(k_{y}\right)\right\rangle$ versus $t$, where blue, green and red curves correspond to the lowest, middle and highest $k_{y}$; and (d) $k_{y}$ spectrum of electrostatic contribution to $q_{e}$ at $t=1200$. For the $\gamma_{E}=0.02$ case in (b): (e) time $(1000<t<2000)$ and field line averaged spectrum of $\left\langle\Phi^{2}\right\rangle\left(k_{x}, k_{y}\right)$; and (f) $\Phi$ contours at the outboard midplane, for $t=2000$. (All times and frequencies are normalised to $a / v_{t e}$ and $v_{t e} / a$ respectively.)

Recent longer ETG simulations, to $t \sim 1500 a / v_{t e}$, with an electrostatic version of the AI model of [7], lead at long times to the runaway growth of $q_{e}$ that is illustrated in Figure 3(a). At late times the $\Phi$ spectrum becomes dominated by a single $k_{x}=0$ 
mode at low $k_{y}$, and this may be associated with a slowly growing mode close to the edge of the spectral domain. A similar phenomenon in previous AI ETG simulations, for a large aspect ratio equilibrium, was cured by including kinetic ions [31], but in our case this merely delayed the onset of the unresolved dominant mode. Figure 3(a) also shows that: including electron collisions (at the experimental level) in the AI model cures the problem and considerably improves the convergence of the spectrum (the same is true for the KI model); and that with a small level of equilibrium flow shear $\left(\gamma_{E}=0.005 v_{t e} / a\right)$ the collisionless AI simulation recovers from what appeared a catastrophically unresolved situation. The healing of the simulation, with the addition either of electron collisions or flow shear, is probably due to the stabilisation of weakly unstable trapped electron driven modes in the region $k_{y} \rho_{i} \sim 1$, as discussed at the end of Appendix A. GYRO simulations have also found that flow shear helps in resolving ETG turbulent saturation with the AI model [32].

The electron heat flux, $q_{e}$, varies by less than a factor 2 in simulations with different perpendicular domain sizes: e.g. the flux tube extent in the binormal direction at the outboard midplane, $L_{y}$, was varied in the range $50<L_{y} / \rho_{e}<100$; and the radial size, $L_{x}$, determined by the parameter $J_{\text {twist }}$ with $L_{x}=J_{\text {twist }} L_{y} /(2 \pi \hat{s})$, was set using $J_{\text {twist }}=2$ and 4 . Linear and nonlinear simulations using both numerical and Miller [33] equilibria give consistent results. Artificial scans in $\gamma_{E} \boldsymbol{\Phi}$, both using initial conditions with low amplitude noise (see Figure 3(b)), and restarting from the saturated state of a resolved simulation with collisions and $\gamma_{E}=0$, find that ETG turbulence is suppressed at $\gamma_{E} \sim 0.05 v_{t e} / a$, which is close to $\gamma_{\max }\left(\gamma_{\max }=0.04 v_{t e} / a\right)$. The steady and well resolved spectrum of $\Phi$ in the AI simulation with electron collisions and $\gamma_{E}=0.005 v_{t e} / a$ is illustrated in Figure 3(c). Figure 3(d) shows the corresponding clearly resolved peak in the $k_{y}$ distribution of the electrostatic contribution to $q_{e}$. The shearing of radially extended ETG streamer structures for the $\gamma_{E}=0.02 v_{t e} / a$ case is evident both in the time and field line averaged spectrum $\left\langle\Phi^{2}\right\rangle\left(k_{x}, k_{y}\right)$, and in the outboard midplane contour plot of $\Phi$, shown in Figures 3(e,f). Turbulence suppression arises at slightly lower $\gamma_{E}$ in fully electromagnetic simulations, where including $\delta \boldsymbol{B}$ gives a lower $\gamma_{\max }=0.025 v_{t e} / a$.

Complementary local simulations were performed with GYRO for MAST-like parameters, including flow shear and KI [34]. These simulations investigated GYRO's non-periodic radial boundary conditions, and demonstrated quantitative convergence with grid size and resolution. GYRO simulations predicted a significant level of electron heat transport, consistent with the above results from GS2. Additional runs demonstrated that ETG transport is sensitive to $q$ and $\hat{s}$, which vary widely across ST plasmas.

In this section we have presented simulations, which run to long times and include the physics of collisions, kinetic ions and sheared toroidal equilibrum flow, that add weight to the conclusion from [7] that ETG turbulence can carry significant electron heat transport at mid-radius in MAST.

I The linear drive term had a negligible impact on ETG turbulence in GYRO simulations for MAST [34], and was excluded from these GS2 studies of the effect of flow shear on ETG turbulence. $q R / r \sim 4$ on this MAST surface, and so the linear drive term should be small. 


\section{Conclusions}

Sheared toroidal equilibrium flows can be sufficiently large to suppress ITG turbulence in STs, and this must significantly affect their energy confinement. Such flows can readily be accommodated within the gyrokinetic framework for $V \sim O\left(M_{\phi} v_{t i}\right)$, for $1>M_{\phi}>\rho_{i} / a$, and affect turbulence through two mechanisms: the sheared perpendicular component of the toroidal flow acts to suppress the transport from microturbulence, but the radial shear in the parallel component of the equilibrium toroidal flow provides a linear drive that can enhance turbulence. The strength of the linear drive relative to the perpendicular shearing term is $\propto(q R / r) B_{\phi} / B$, and this factor is relatively small at mid-radius in the ST. We can therefore expect STs to provide a favourable environment for turbulence suppression through sheared toroidal flow.

Sheared toroidal flow has recently been implemented and tested in two local flux-tube gyrokinetic codes (GS2 and GKW). Linear gyrokinetic simulations using GS2, including equilibrium flow shear at the approximate level realised in the MAST experiment, demonstrate that ITG modes can indeed be suppressed while ETG modes remain unstable. ETG turbulence simulations have been presented for conditions typical of mid-radius in MAST, and show that both collisions and modest equilibrium flow shear can sharpen the low $k_{y}$ cut-off to significantly improve the resolution of the saturated turbulence. In numerical experiments with artificially raised levels of $\gamma_{E}$, it is found that the ETG turbulence only starts to be diminished as $\gamma_{E}$ approaches the value of the maximum linear ETG growth rate. These results add weight to the main conclusion of [7] that ETG induced transport can be significant at mid-radius in MAST.

Flows in MAST are close to sonic, with $M_{\phi} \sim 0.4$, and to improve current GK simulations it will be necessary in the future to include self-consistently both the Coriolis force (which is included in GKW [35]) and the centrifugal force.

\section{Acknowledgments}

We are very grateful to Alberto Bottino, Jeff Candy, Joachim Hein, Gavin Pringle, Gary Staebler, Ron Waltz and Michele Weiland for many helpful discussions, and to DEISA and EPSRC for access to the supercomputers BABEL and HECToR, where some of the reported simulations were carried out. This work was funded partly by the United Kingdom Engineering and Physical Sciences Research Council and by the European Communities under the contract of Association between EURATOM and UKAEA, in part by the Leverhulme Trust Network for Magnetised Plasma Turbulence, and A Schekochihin was partly supported by STFC. The views and opinions expressed herein do not necessarily reflect those of the European Commission.

\section{References}

[1] R. J. Akers et al., Transport studies in the MAST spherical tokamak, in Proceedings of 22nd IAEA Fusion Energy Conference, Geneva, EX2-2, 2008.

[2] S. M. Kaye et al., Nuclear Fusion 47, 499 (2007).

[3] H. Biglari, P. H. Diamond, and P. W. Terry, Physics of Fluids B 2, 1 (1990).

[4] R. E. Waltz, G. D. Kerbel, and J. Milovich, Physics of Plasmas 1, 2229 (1994). 
[5] M. Kotschenreuther, G. Rewoldt, and W. M. Tang, Computer Physics Communications 88, 128 (1995).

[6] A. G. Peeters and D. Strinzi, Physics of Plasmas 11, 3748 (2004).

[7] N. Joiner et al., Plasma Physics and Controlled Fusion 48, 685 (2006).

[8] M. Wakatani et al., Nuclear Fusion 39, 2175 (1999).

[9] M. Valovic et al., Nuclear Fusion 49, 075016 (2009).

[10] A. R. Field et al., Core Heat Transport in the MAST Spherical Tokamak, in Proceedings of 20th IAEA Fusion Energy Conference, Vilamoura, Portugal, EX/P2-11, 2004.

[11] C. M. Roach et al., Nuclear Fusion 48, 125001 (2008).

[12] G. M. Staebler, J. E. Kinsey, and R. E. Waltz, Physics of Plasmas 12, 102508 (2005).

[13] G. M. Staebler et al., Testing the Trapped Gyro-Landau Fluid Transport Model with Data from Tokamaks and Spherical Tori, in Proceedings of 22nd IAEA Fusion Energy Conference, Geneva, TH/P8-42, 2008.

[14] S. Jolliet et al., Computer Physics Communication 177, 409 (2007).

[15] J. Candy and R. E. Waltz, Journal of Computational Physics 186, 545 (2003).

[16] D. J. Applegate, C. M. Roach, and S. C. Cowley et al, Physics of Plasmas 11, 5085 (2004).

[17] C. M. Roach et al., Plasma Physics and Controlled Fusion 47, B323 (2005).

[18] D. J. Applegate et al., Plasma Physics and Controlled Fusion 49, 1113 (2007).

[19] L. Vermare et al., Journal of Physics Conference Series 123, 012040 (2008).

[20] D. Told et al., Physics of Plasmas 15, 102306 (2008).

[21] K. L. Wong et al., Physics of Plasmas 15, 056108 (2008).

[22] D. Stutman et al., Physical Review Letters 102, 115002 (2009).

[23] M. Artun and W. M. Tang, Physics of Plasmas 1, 2682 (1994).

[24] G. W. Hammett et al., Implementation of Large Scale ExB Shear Flow in the GS2 Gyrokinetic Turbulence Code, in American Physical Society, 48th Annual Meeting of the Division of Plasma Physics, abstract VP1.136, 2006.

[25] J. W. Connor, J. B. Taylor, and H. R. Wilson, Physical Review Letters 70, 1803 (1993).

[26] R. E. Waltz, R. L. Dewar, and X. Garbet, Physics of Plasmas 5, 1784 (1990).

[27] J. Kinsey, R. E. Waltz, and J. Candy, Physics of Plasmas 12, 062302 (2005).

[28] A. M. Dimits et al., Physics of Plasmas 7, 969 (2000).

[29] A. G. Peeters and C. Angioni, Physics of Plasmas 12, 072515 (2005).

[30] N. J. Joiner, Microinstabilities in Spherical Tokamaks, PhD thesis, Imperial College, London, 2005.

[31] W. M. Nevins et al., Physics of Plasmas 13, 122306 (2006).

[32] J. Candy et al., Plasma Physics and Controlled Fusion 49, 1209 (2007).

[33] R. L. Miller et al., Physics of Plasmas 5, 973 (1998).

[34] W. Guttenfelder et al., Gyrokinetic Simulations of Electron Scale Turbulence in Spherical Tokamak Plasmas with Flow Shear, in EPS, Sofia, 2009.

[35] A. Peeters, C. Angioni, and D. Strintzi, Physical Review Letters 98, 265003 (2007).

\section{Appendix A. Comparing Electrostatic Microstability Analyses from Local GK Codes for a MAST Equilibrium}

We have compared linear results from the local gyrokinetics codes GKW, GS2 and GYRO, using parameters of a strongly shaped equilibrium flux surface in the MAST H-mode discharge \#8500 [10,17]: $\rho=r / a=0.7, r / R=0.443, q=2.1, \hat{s}=(\rho / q)(d q / d \rho)=2.2$, $\beta=0.06, T_{i}=0.385 \mathrm{keV},\left(1 / T_{i}\right) d T_{i} / d \rho=-4.39, T_{e}=0.346 \mathrm{keV},\left(1 / T_{e}\right) d T_{e} / d \rho=-2.79$, $n_{e}=5.7 \times 10^{19} \mathrm{~m}^{-3},\left(1 / n_{e}\right) d n_{e} / d \rho=-0.44$, elongation $\kappa=1.56, d \kappa / d \rho=0.5$, triangularity $\delta=0.2, d \delta / d \rho=0.43$, and $(1 / a) d R / d \rho=-0.29$. Figure A1(a) demonstrates good agreement between the computed spectra of the dominant linear growth rate for a collisionless electrostatic model with kinetic electrons and without flow shear.

Figure A1(b) shows, from GS2 runs using a local Miller parameterisation of the 


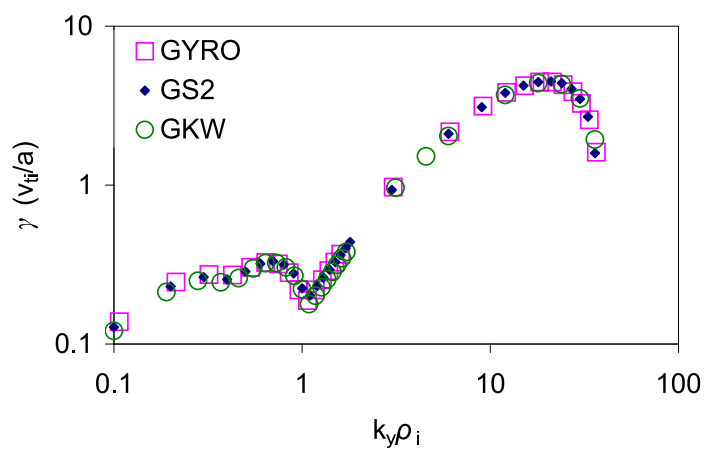

a

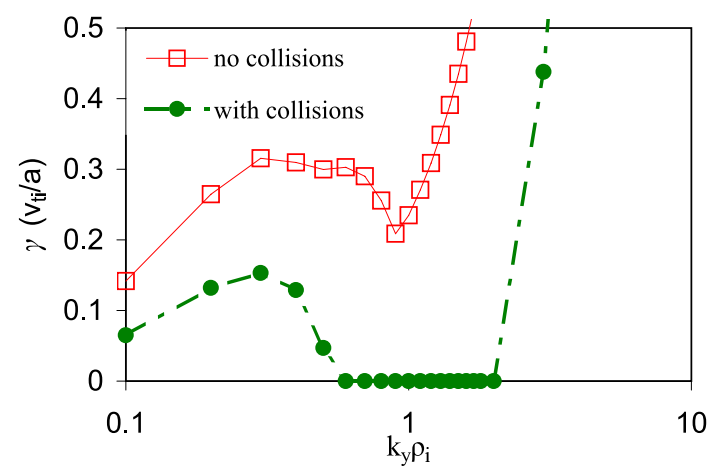

$\mathrm{b}$

Figure A1. $\gamma$ normalised to $v_{t i} / a$ versus $k_{y} \rho_{i}$ for linear electrostatic calculations with kinetic electrons for the MAST equilibrium described: (a) without collisions from GS2, GKW and GYRO and (b) with and without collisions from GS2.

equilibrium [33], that with collisions the modes around $k_{y} \rho_{i} \sim 1$ become stable. Estimating the ratio of the electron detrapping frequency to the diamagnetic frequency, for these parameters, gives $\nu_{\text {detrap }}^{e} / \omega_{*} \geq 1$ for $k_{y} \rho_{i} \sim 1$, which is consistent with a reduced trapped electron drive around this wavenumber. 